\title{
Resistive Fault Current Limiters with YBCO Films - 100 kVA Functional Model
}

\author{
B. Gromoll, G. Ries, W. Schmidt, H.-P. Kraemer, B. Seebacher, B. Utz, R. Nies and H.-W. Neumueller, \\ Siemens AG, Corporate Technology, Erlangen, Germany
}

E. Baltzer, S. Fischer, Siemens AG, Power Transmission and Distribution, Berlin, Germany

B. Heismann, Friedrich-Alexander-University, Erlangen, Germany

\begin{abstract}
Within the Siemens current limiter program a 100 kVA functional model has been realized and tested successfully. According to the modular concept of the limiter the functional model is assembled of ten switching elements. YBCOfilms with a thickness of $250 \mathrm{~nm}$ and a critical current density above $2 \times 10^{6} \mathrm{~A} / \mathrm{cm}^{2}$ are deposited by thermal coevaporation (TU Munich) on 4" sapphire wafers. To support homogeneous switching the superconducting film is covered with a $100 \mathrm{~nm} \mathrm{Au}$ - shunt layer. Good current limiting performance is achieved: The steady limiting current is below the nominal current, the peak fault current increases up to 3 times the nominal current within an action time of one millisecond. The operational recovery time of the limiter, within which the switching elements return to the superconductive state again is about $2 \mathrm{~s}$. Following our limiter program the next step will be a model device with a nominal switching power of 1 MVA.
\end{abstract}

\section{INTRODUCTION}

In an electric power system the task of Fault Current Limiters (FCL) is the limitation of mechanical and thermal loads on busbars, insulators and circuit breakers during a short circuit. Several types of FCL concepts based on superconducting materials have been proposed so far, an overview is given in [1]. A 1.2 MVA prototype of the inductive "shielded iron core" type has been built and tested by ABB [2]. The so-called resistive FCL relies on the switchlike onset of resistivity during a quenching process caused by exceeding the superconductor's critical current. This results in a self-triggered limitation of a fault-current passed through the device and the FCL therefore works as a fast and selfrestoring fuse with passive triggering. Applications range from new systems to upgrading existing networks with increased fault currents, or it may be used as an integrated component of superconductive systems like cables, transformers and SMES or in the feed line of power generators.

Manuscript received September 14, 1998

This work has been supported by the German Federal Ministry for Education, Science, Research and Technology BMBF under contract no. 13N6842A.

Part of the work is done within the joint collaboration between Hydro Quebec, Canada and Siemens, Germany.
Based on the results of our $20 \mathrm{kVA}$ limiter [3] this paper reports the implementation and successful testing of a $100 \mathrm{kVA}$ FCL functional model at Siemens Erlangen.

\section{SETUP OF THE $100 \mathrm{KVA}$ FUNCTIONAL MODEL}

In our modular setup of the functional model, ten switching elements are assembled to achieve the overall nominal switching power of $P_{\text {nom }}=100 \mathrm{kVA}$. Each of them consists of an YBaCuO film with a thickness of $250 \mathrm{~nm}$ deposited on 4" sapphire wafers by means of thermal coevaporation (TU Munich [4]). The resistively measured critical currents $I_{c}$ of the switching elements vary between $30 \mathrm{~A}$ and $48 \mathrm{~A}$. Inductive scans of the critical current density show that the spatial variations within one element are significantly less. Since these inhomogeneities can cause the formation of hot spots during switching the $\mathrm{YBaCuO}$ film is

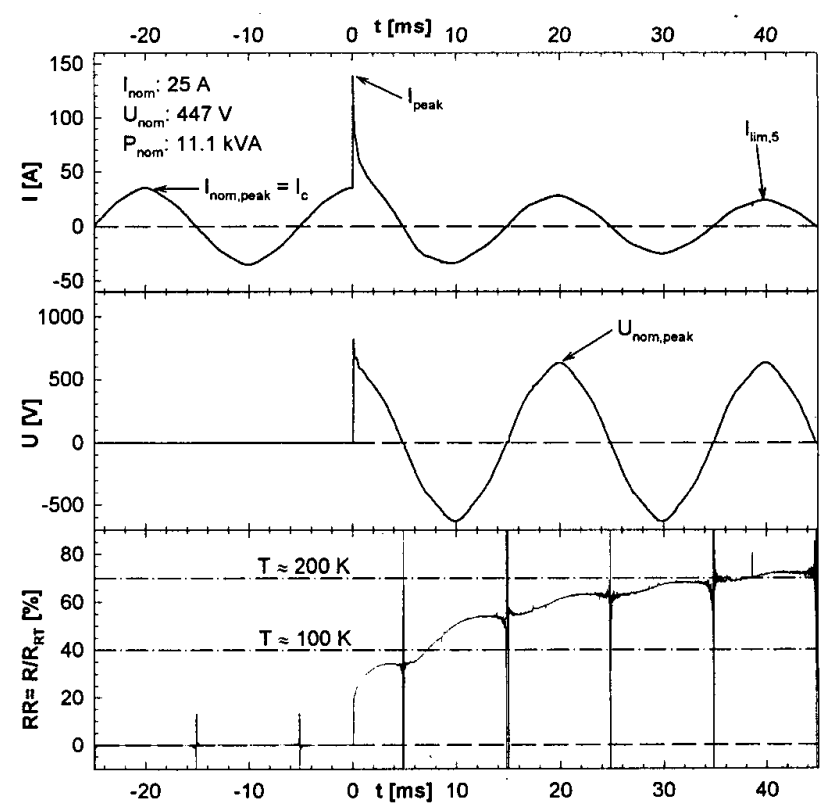

Fig. 1: Plots of current, voltage and resistance during the switching behavior measurement of a single sample 
TABLE I

DATA OF STACK CONFIGURATION

$\begin{array}{lccc} & \mathrm{I}_{\mathrm{c}}[\mathrm{A}] & \mathrm{I}_{\text {peak }}[\mathrm{A}] & \mathrm{I}_{\text {lims }}[\mathrm{A}] \\ \text { Stack 1 } & 189.2 & 667.9 & 107.7 \\ \text { Stack 2 } & 196.7 & 666.2 & 108.2\end{array}$

covered with an additional $100 \mathrm{~nm}$ gold shunt layer. A spiral strip with a length of $80 \mathrm{~cm}$ and a path width of $7 \mathrm{~mm}$ was etched into the layer structure.

At first the switching elements were tested individually. They were bath-cooled in a cryostat filled with liquid nitrogen and exposed to short circuit conditions simulated in the laboratory. After carrying the nominal current $I_{\text {nom }}=I_{c} / \sqrt{ } 2$ for some AC-cycles, they were facing the short-circuit starting in the middle of a half-cycle and persisting another four AC half-cycles, i.e. $\Delta t=45 \mathrm{~ms}$ in total. Fig. 1 shows the typical current, voltage and resistance behavior of a single sample as a function of time. The resulting peak let-through current $I_{\text {peak }}$ and the limited current $I_{\text {lim,s }}$ in the first and fifth half cycle were taken to characterize the switching behavior. The resistance normalized to its room temperature value $R R$ is used as an indication of the sample temperature. In the measurement with a nominal power of $11 \mathrm{kVA}$ shown in Fig. 1 the temperature reaches a maximum near $200 \mathrm{~K}$.

In order to achieve $P_{\text {nom }}=100 \mathrm{kVA}$ with a voltage below the available maximum of $1000 \mathrm{~V}$ the ten elements were arranged as a series of two stacks each consisting of five

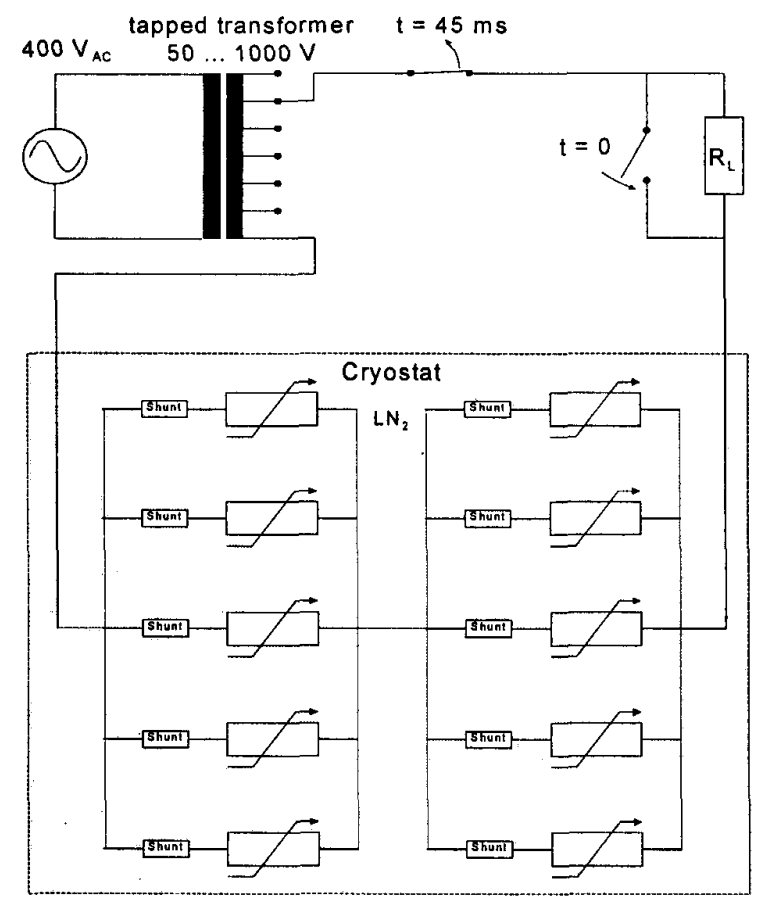

Fig. 2. Schematic setup of the $100 \mathrm{kVA}$ functional model and the test circuit

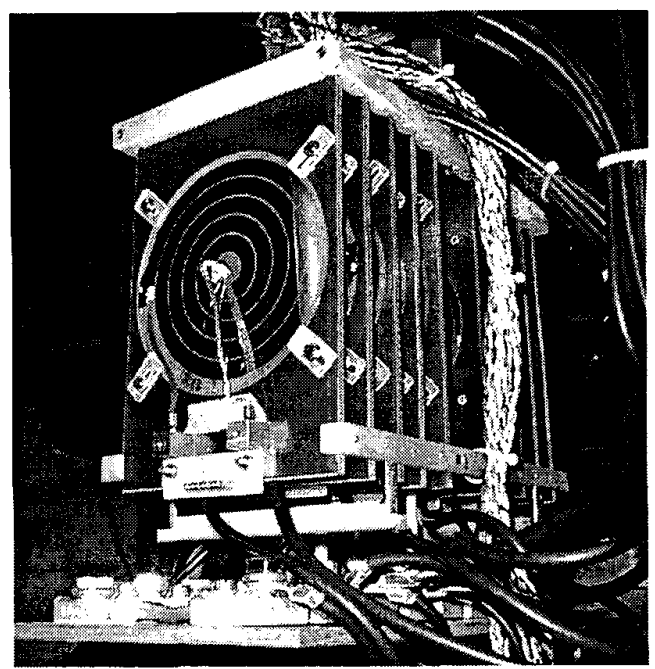

Fig. 3: Photo of the assembled switching elements and the corresponding shunt resistors.

elements in parallel. For the selection of the elements the following criteria were adopted:

- Obviously, the sum of critical currents $I_{c}$ in both stacks should be equal, because the lower value determines the nominal current of the device.

- Since $I_{\text {peak }}$ reflects the speed of the resistive onset in an individual element and homogenous switching is essential, the sums of the peak currents should be the same as well.

- Finally, the limited currents $I_{\text {lim, }}$ should also be equal to ensure that each of the stacks is exposed to approximately the same voltage during the current limitation.

As shown in Table I the chosen configuration leads to a nominal current of $I_{\text {nom }}=189 \mathrm{~A} / \sqrt{2}=135 \mathrm{~A}$ and sums of $I_{\text {peak }}$ and $I_{\text {lim, } 5}$ that correspond up to less than $1 \%$ deviation between both stacks. Fig. 2 depicts the experimental setup of both the switching elements and the circuitry used to test them. Each switching element is supplied with a $1 \mathrm{~m} \Omega$ shunt resistance in order to be able to measure the individual current. Photograph in Fig. 3 shows the positioning of the samples and the assembly of the shunts below the sample holders. The spacing between the elements is $2 \mathrm{~cm}$ and the overall dimensions of the ten elements are $22 \mathrm{~cm} \mathrm{x} 15 \mathrm{~cm} \mathrm{x}$ $12 \mathrm{~cm}$.

\section{TEST OF 100 KVA LIMITING CAPABILITY}

With the stacks put in series, short-circuit experiments with increasing nominal voltages $U_{\text {nom }}$ were performed. At a setting of $750 \mathrm{~V}$, the switched nominal power reached $P_{\text {nom }}=765 \mathrm{~V} \cdot 135 \mathrm{~A}=103 \mathrm{kVA}$. Fig. 4 depicts the measured current and voltage behavior. $I_{\text {peak }}$ was measured to be 3 times the peak nominal current, while the limited current $I_{\text {lim, } 5}$ amounted to $65 \%$ of $I_{\text {nom,peak. }}$. The resistance ratio RR was up 


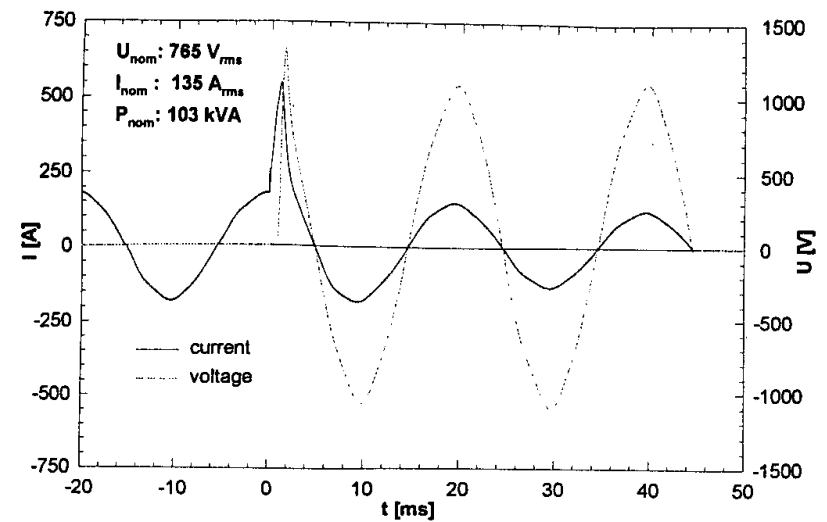

Fig. 4. Current and voltage as function of time during a $100 \mathrm{kVA}$ limiting experiment

to $72 \%$ of the room temperature resistance. The experiment was repeated 8 times at a nominal power of $100 \mathrm{kVA}$ without any damage or degradation.

The top diagram of Fig. 5 shows the distribution of the transient voltages $U_{1}, U_{2}$ and dissipated energies $Q_{1}, Q_{2}$ between the two stacks during the $100 \mathrm{kVA}$ tests. At the end
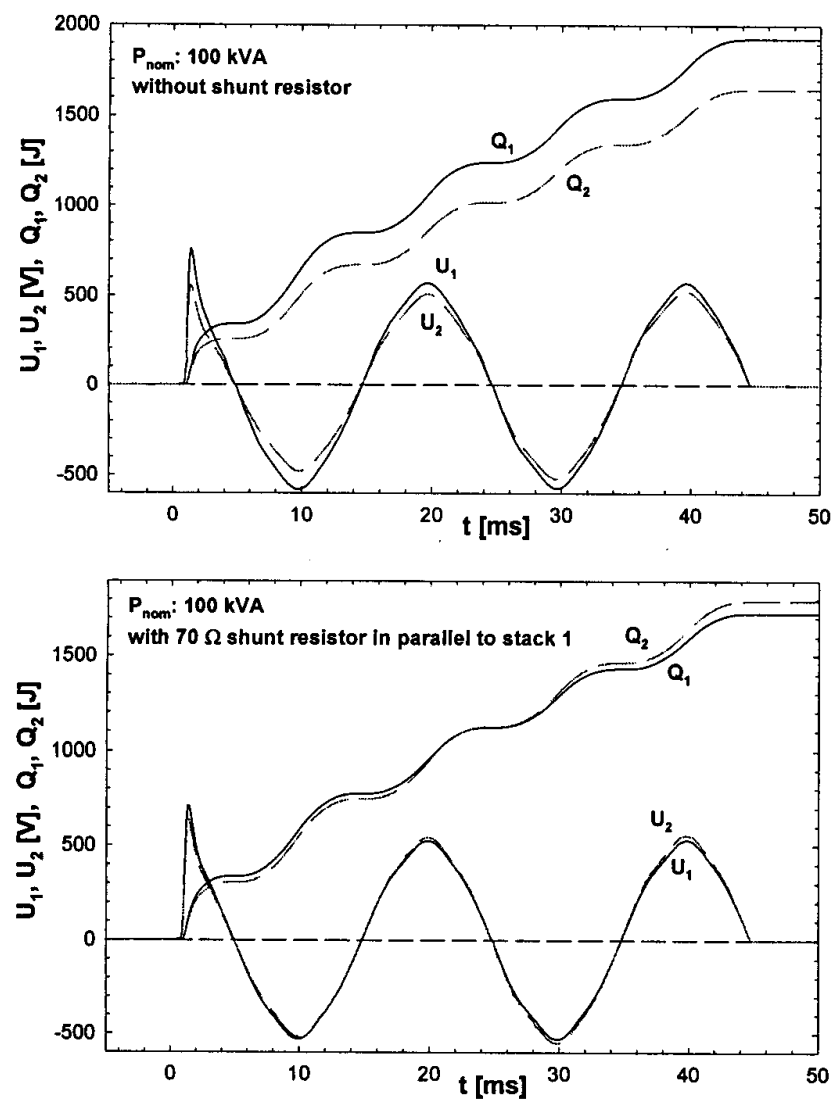

Fig 5. Plots of voltages and deposited energies of the two stacks during a $100 \mathrm{kVA}$ switching experiment, top: without shunt resistor, bottom: with 70 $\Omega$ shunt resistor parallel to stack 1 of the limiting phase the dissipated energy $Q_{1}$ of stack 1 exceeds $\mathrm{Q}_{2}$ by $17 \%$. In our configuration voltage redistributions between the stacks put in series and rapid current redistributions between parallel samples indeed have a very complex behavior. Nevertheless, apart from the initial balancing of the limited and the peak currents, this impact of inhomogeneity can be reduced by adding a parallel resistance to one of the stacks since the current passed through it and therefore the evolving resistance is reduced. In this case, a shunt resistance of $70 \Omega$ in parallel with stack 1 leads to a reduced imbalance, the result is shown in the bottom of Fig. 5: the voltage and current curves of both stacks are very close. Further we found that the difference between the maximum and minimum energy deposited on a single element is roughly cut in half. This not only decreases the maximum load on a single element, but also permits a faster recooling of the whole FCL setup.

\section{TRANSIENT CURRENT REDISTRIBUTIONS}

As an interesting feature of the $100 \mathrm{kVA}$ measurements rapid current redistributions during the phase transition of parallel components of a single stack were observed. In detail this has been studied using a parallel configuration of four of the ten elements with $I_{c}$ values between $30 \mathrm{~A}$ to $48 \mathrm{~A}$. Fig. 6 indicates the evolution of the current through each sample during a short circuit experiment. Up to $I(t)=50 \mathrm{~A}$, the resistivities of the normal-conducting interconnections dominate and according to this each sample carries the same current. Beyond $50 \mathrm{~A}$ the flux-creep resistivity leads to a slow redistribution of the individual currents: element $\mathrm{C}$ with the highest $I_{c}$ carries the highest, element $D$ with the lowest $I_{c}$ the lowest current. This seems to be a consequence of the flux-creep resistivity being proportional to $\left(\mathrm{j} / \mathrm{j}_{c}\right)^{\mathrm{n}}$. But on the scale of a whole switching element this only turns out to be a

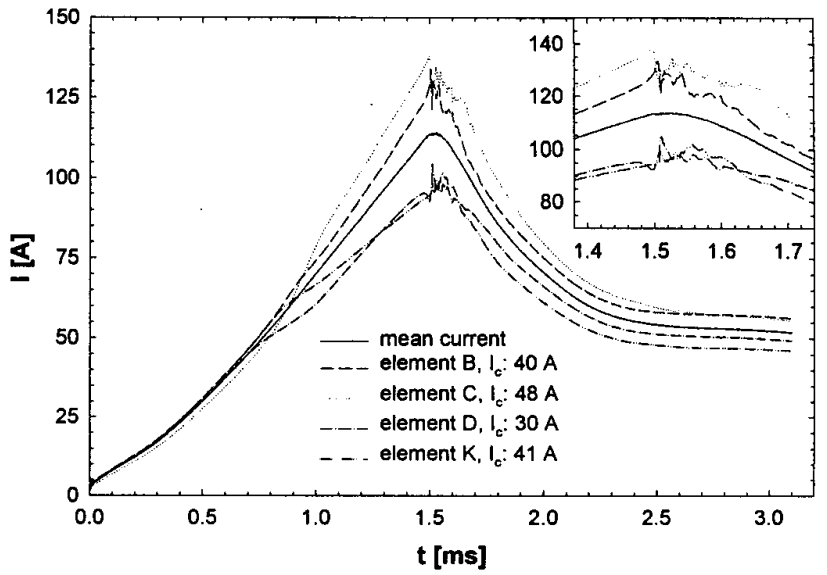

Fig. 6. Transient current redistribution during a $42 \mathrm{kVA}$ switching experiment, four switching elements in parallel 
tendency as can readily be seen from samples $B$ and $K$ which have almost the same $I_{c} \approx 40$ A but carry noticeable different transient currents.

After about $1.5 \mathrm{~ms}$ the normal state resistivity sets in and leads to high-frequency current redistributions between the samples, see also the inset of Fig. 6. The calculated mean of the four current traces shows the expected smooth behavior. Interestingly, this rapid adaptation of the currents to the fluxcreep and flux-flow-resistivities should prevent an ensemble of parallel samples from a premature burn-out since the elements are forced to enter the normal phase resistive state at the same time. Because of the currents preferring the paths with lower flux-creep and flux-flow resistance, the power dissipation tends to be distributed equally.

\section{OPERATIONAL RECOVERY TIME}

After $45 \mathrm{~ms}$ of Ohmic heating the deposited Joule energy is mainly stored in the substrate of a switching element. Its cooldown via heat transfer into the nitrogen bath takes place within the order of seconds. The operational recovery time $\tau_{r}$ defines the minimum cooldown time after which the FCL can carry the nominal current again without quenching.

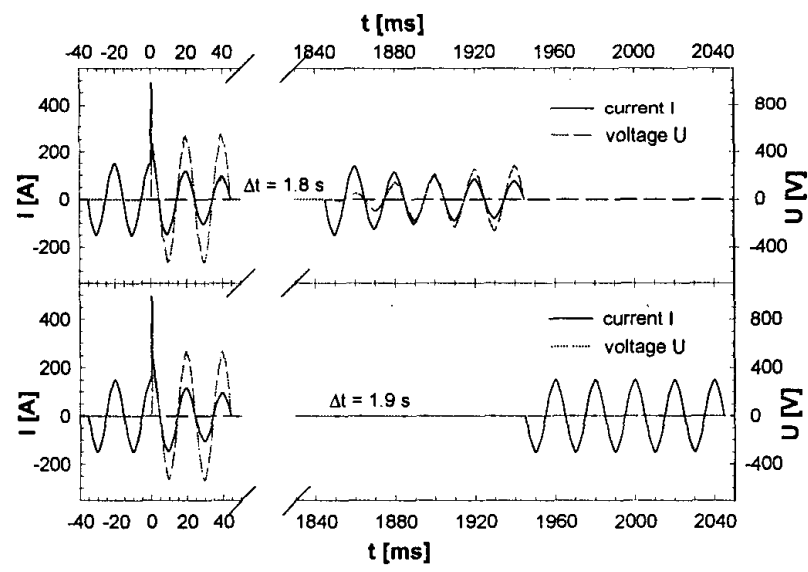

Fig. 7. Current and voltage during reactivation of the limiter before (top) and after (bottom) the switching elements have cooled down completely.

Using the same stack of four elements in parallel, at a nominal switching power of $42 \mathrm{kVA} \tau_{\mathrm{r}}$ was measured to be 1.9 s. Fig. 7 (top) shows the voltage and current evolution when the nominal current is switched on again after $1.8 \mathrm{~s}$, i.e. before all the elements have cooled down completely. In this case the limiter quenches again. The bottom curves in Fig. 7 prove that after a cooldown time of $1.9 \mathrm{~s}$ the limiter can carry the full nominal current without any voltage drop.

\section{CONCLUSION}

It has been demonstrated that with high $\mathrm{j}_{\mathrm{c}}$ YBCO films on sapphire substrates it is possible to realize a very efficient and compact fault current limiter of the $100 \mathrm{kVA}$ class. The modular concept makes it possible to utilize switching elements with a considerable bandwidth of critical currents. The fast current redistribution at the beginning of the switching phase prevents an ensemble of parallel elements from burn-out. The functional model reached a power density, i.e. the nominal power per substrate area, of about $130 \mathrm{~W} / \mathrm{cm}^{2}$. Experiments with single switching elements and recent results of other groups [5] indicate that the maximum achievable power density is much higher. This would make the devices even more compact. In the near future we will produce switching elements of $20 \mathrm{~cm} \mathrm{x} 20 \mathrm{~cm}$ and build up a 1 MVA FCL model.

\section{REFERENCES}

[1] R.F. Giese and M. Runde, "Fault Current Limiters," Argonne National Laboratory, November 1, 1991

R.F. Giese and M. Runde, "Fault Current Limiters-A Second Look," Argonne National Laboratory, March 16, 1995

[2] W. Paul, M. Lakner, J. Rhyner, P. Unternaehrer, Th. Baumann et al., "Test of 1.2 MVA High- $T_{c}$ Superconducting Fault Current Limiter," Applied Superconductivity 1997, vol. 2, p. 1173-1178, Institute of Physics Publishing, Bristol and Philadelphia, 1997

[3] B. Gromoll, G. Ries, W. Schmidt, H.P. Kraemer, B. Seebacher et al., "Resistive Current Limiters with YBCO Films," Applied Superconductivity 1997, vol. 2, p. 1243-1246, Institute of Physics Publishing, Bristol and Philadelphia, 1997

[4] H. Kinder, W. Prusseit, R.Semerad, B. Utz et al., "YBCO Deposition on Large Substrates up to 9" in Diameter," Adv. in Siperconductivity IX (ISS '96), vol. 2, p. 1011-1016, Springer-Verlag, Tokyo, Japan, 1997

[5] A. Heinrich, H. Kinder, H. Mosebach, "Fault Current Limiting Properties of YBCO-Films on Sapphire Substrates," unpublished, submitted to ASC ' 98 